\title{
Design of P2P-SIP System Network Structure Based on JXTA
}

\author{
Lei Shi, Chen Shi, Hongying Wang* \\ Henan Polytechnic Institute, Nayang, Henan, China \\ *Corresponding Author.
}

\begin{abstract}
Session Initiation Protocol (SIP) is one of the most important signaling connection protocols in the field of VoIP (Voice over Internet Protocol). However, the existing SIP network has some problems such as manual configuration, difficult maintenance and poor scalability. With the development of P2P (Peer-to-Peer) technology, the combination of SIP and P2P has become a trend. JXTA is an open peer-to-peer platform, which provides basic application services for building P2P network. The topic chooses JXTA development platform to build P2P network, and then constructs SIP soft terminal on it. Based on the in-depth study of JXTA structured network and SIP protocol, a P2P SIP system is designed and implemented. The system can maintain normal SIP communication without maintaining the central server. In this paper, the detailed design and implementation process of P2P-SIP system are given, including node join and leave, user join, user search and session establishment. This paper focuses on the implementation process of user search, that is, the collaborative process of discovery service, resolution service, aggregation service and endpoint routing service. Finally, the feasibility of the system is verified by functional test.
\end{abstract}

Keywords: Session Initiation Protocol, P2P, QoS, JXTA

\section{Introduction}

In recent years, VoIP is gradually replacing the traditional telephone system and becoming a hot application of communication network. Session initiation protocol (SIP) developed by IETF adopts the traditional structure of $\mathrm{C} / \mathrm{S}$ (client / server) and relies on the central server, which has the problems of "single point failure" and "performance bottleneck" [1-2]. There is no central server in P2P (peer-to-peer) network, which realizes the direct communication between nodes in a decentralized self-organization way. The participants in the network are not only resource providers, but also resource takers.

The combination of P2P and SIP is one of the hot issues in current application research. The SIP system based on $\mathrm{P} 2 \mathrm{P}$ technology (P2P-SIP) combines the concepts of self-organization and distribution in P2P network structure into sip, and searches distributed resources in SIP network, so as to realize the system structure separated from centralized server.

Generally speaking, there are two main technical routes to combine P2P and SIP technology. The first one is to extend SIP and use existing SIP to carry P2P mechanism. Bryan conceived a P2P-SIP solution SOSIMPLE based on Chord, which removed the SIP central server entity and required extended SIP and specific Chord support. Singh, Schulzrinne, etc. provided an open implementation scheme of Internet telephony client adapter SIPPEER based on P2P-SIP. This design is based on DHT P2P structure and carries the registration and discovery of P2P mechanism through SIP messages [3]. The second scheme of combining P2P and SIP is to apply P2P mechanism to user discovery and realize P2P-based location service. Compared with the protocol extension mode, the second scheme maintains the compatibility with standard SIP, only the publishing and discovery process is based on P2P technology, and the session signaling processing process is still traditional SIP [4]. HolgerSchmidt [5], Burcin [6] and others [7-9] have put forward a P2P-SIP implementation scheme based on this technical route, in which P2P mechanism is built into proxy server, and P2P network is formed at proxy server level to realize message transmission. This architecture does not completely break away from the role of SIP proxy server.

ISSN: 0010-8189 


\section{Technical background of P2P-SIP system}

\subsection{SIP}

Standard SIP structure entities include user agent (UA), register server, proxy server and redirection server. The user agent registers the user and his contact address with the registration server [10-11]. The registration information enters the public location server of IP network. The SIP server searches the user's location by accessing the server. The redirection server is responsible for returning the routing information to the call initiator. After receiving the invite request from the caller, the redirection server locates the destination address and returns it to the caller. After that, the caller can send a new request to the address returned by the server to establish a connection. The proxy server is responsible for routing messages between user agents, forwarding the requests sent by the caller to other servers or terminals, and returning the responses sent back by the server to the terminal. The caller initiates a session by sending an invite request to the proxy server, which is forwarded by other servers in the network, and finally arrives at the called user agent to complete the call establishment process of three handshakes.

\subsection{JXTA}

JXTA is an open network computing platform based on P2P technology designed by Sun company, which can realize the wide application of P2P. JXTA consists of six protocols, which allow all connected devices (including cellular phone, PDA, PC and server) in the network to communicate and cooperate as nodes. Each resource in JXTA network is called peer, and multiple peers form a peer group. Peer can be divided into edge peer and aggregate peer. The aggregated peers form a ring topology. Each peer has a unique ID and can be represented by an advertisement. The process of peer discovery is the process of searching JXTA network for the node's announcement, which is realized by sending broadcast messages and aggregating peer index in JXTA network.

\section{Design of P2P-SIP based on JXTA}

This paper proposes a new implementation scheme of P2P-SIP system based on JXTA platform, which uses P2P publishing and location mechanism to replace the registration and search process of standard sip. This design is completely separated from SIP proxy server, facing user agent UA, forming P2P network at UA level. Firstly, JXTA platform is built as the basic platform to realize $\mathrm{P} 2 \mathrm{P}$ application mechanism, and a $\mathrm{P} 2 \mathrm{P}$ extension module (p2pext) is built by using JXTA platform interface to realize the publish and locate functions of P2P-SIP based on JXTA. We improve the standard SIP client to peerclient, and the P2P extension module provides the communication interface between peerclient and JXTA domain, so that the client can control the publishing and positioning behavior, so as to combine the $\mathrm{P} 2 \mathrm{P}$ mechanism with the standard sip. Users can publish user resource announcements in the network through publishing function. The location function can directly find and find the target users in JXTA network without routing through the proxy server, so as to realize the network structure separated from the proxy server. The peer client and the P2P extension module are placed in the same user terminal, each user terminal has an independent contact address (the IP address of the user host), and each user is identified in the form of sip-uri. Any user resource can publish announcements in JXTA network, and can find announcements published by other users in peer-to-peer network. The user resource announcement contains several fields to identify the information and services required by the user. The name field represents the sip-uri of the user, and the desc field represents the contact address of the user. The user initiates the publishing behavior by sending the publishing request to the P2P extension module. After receiving the publishing request from the local peer client, the P2P extension module publishes the user's resource announcement in the JXTA network. Before a user initiates a call, he sends a location request to the P2P extension module. After receiving the location request from the local peerclient, the P2P extension module obtains the sip-uri of the target user and sends a broadcast message in the JXTA network. Search for the notice that the name field matches the sip-uri, find the notice of the target user and return it to the client. The client extracts the contact address of the target user from the desc field, directly initiates the invite call request to the target user, and stores the found notice in the local cache for later use. The process is shown in Figure 1.

ISSN: 0010-8189 


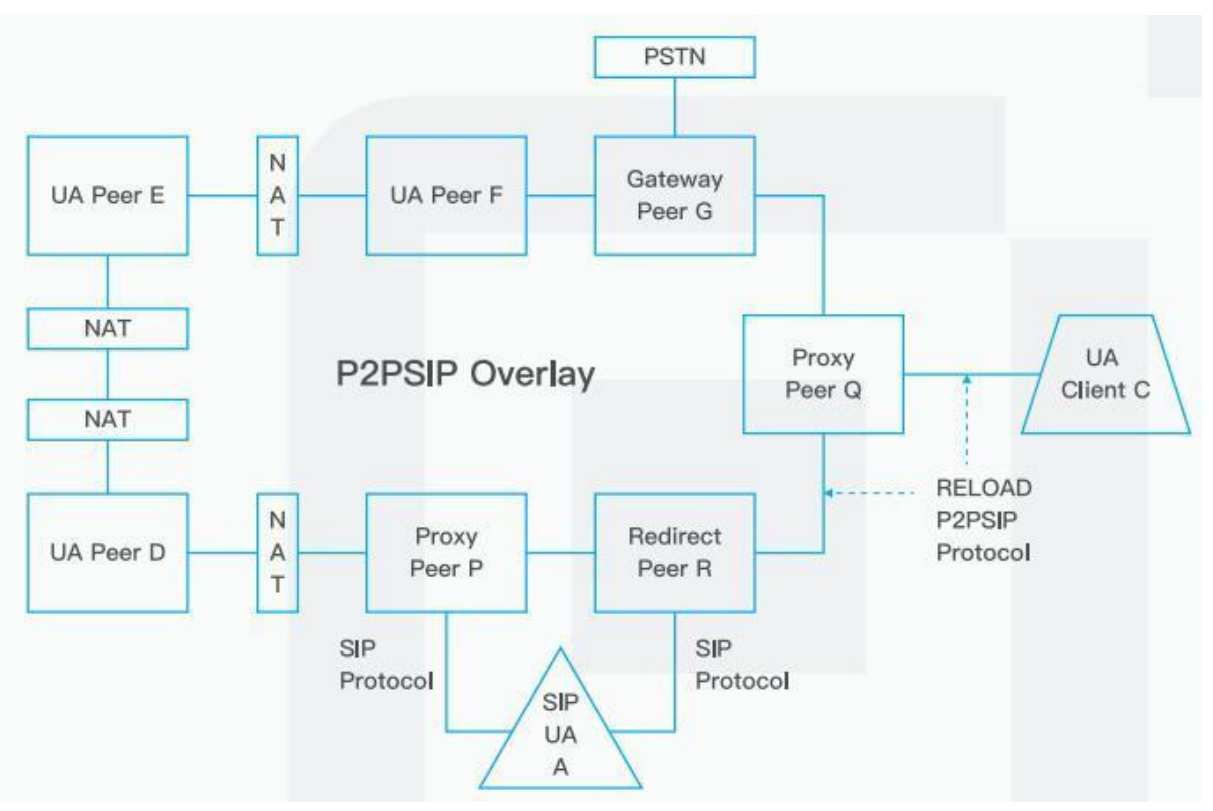

Fig 1: P2P-SIP based on JXTA

\subsection{Functional structure}

The designed P2P-SIP system mainly includes peerClient, SIP module and P2P extension module. P2P extension module realizes the publishing and positioning mechanism of P2P network, SIP module provides session signaling to realize call service, and client is the user's operation interface. P2P module is divided into Access module and Adapter module. In this invention, the open source SIPstacks are directly adopted to realize the call signaling function of SIP, and on the basis of supporting the standard SIP client program, the original registration and call interface functions are replaced by the publishing and positioning function interface functions to realize the intercommunication with Access. Access is the interface between client and P2P module, and Adapter is functionally equivalent to a P2P server, which controls the publishing and positioning mechanism of users based on JXTA. P2P-SIP interface protocol JXP is defined between Access and Adapter. The detailed module structure of P2P-SIP is shown in Figure 2.

\begin{tabular}{|c|c|c|c|c|}
\hline & & & RTCPeer & DataChannel \\
\hline XHR & SSE & WebSocket & & SCTP \\
\hline \multicolumn{3}{|c|}{ HTTP 1.x/2.0 } & \multicolumn{2}{|c|}{ Session (DTLS) - mandatory } \\
\hline \multicolumn{3}{|c|}{ Session (TLS) - optional } & \multicolumn{2}{|c|}{ ICE, STUN, TURN } \\
\hline \multicolumn{3}{|c|}{ Transport (TCP) } & \multicolumn{2}{|c|}{ Transport (UDP) } \\
\hline \multicolumn{5}{|c|}{ Network (IP) } \\
\hline
\end{tabular}

Fig 2: P2P-SIP module structure

ISSN: 0010-8189 


\subsection{JXP data unit definition format}

This design defines JXP between access and adapter, which negotiates the request specification for publishing and locating local domain and JXTA domain. In the design, JXTA notification is used to record a user's URI and the IP address of the login terminal. JXP is mainly used in two aspects: one is the publication of notices in JXTA network; the other is the query of needed notices in JXTA network. The content of JXP defined is shown in Table 1 .

Table 1 Protocol format of JXP

\begin{tabular}{|c|c|c|c|c|}
\hline TYPE & URI SIZE & URI & IP SIZE & IP \\
\hline 1 byte & 2 byte & Any length & 2 byte & Any length \\
\hline
\end{tabular}

The functions of each field are as follows :

TYPE field: indicates the type of request, 0 indicates the publishing request, and 2 indicates the positioning request.

URI SIZE field: it can be used to publish requests and locate requests, and store the uri length.

URI field: it can be used for publishing request and positioning request. when used as publishing request, it stores user URI here, and when used as positioning request, it is destination user URI here.

IP SIZE field: used to locate the request and store the IP address length of the login terminal.

IP field: used for positioning request and storing the IP address of login terminal.

\section{Signaling example}

In the following example, it is assumed that a simple P2P network is used, which is called netsim and has users A and B. The URI of user a is 1000@room1.netsim, and the IP address of the terminal is 10.10.80.2; The URI of user b is $1001 @$ room1.netsim, and the IP address of the terminal is 10.10 .80 .124 . Users a and b log in on the client and publish announcements in JXTA network. the process of publishing events is shown in Figure 3.

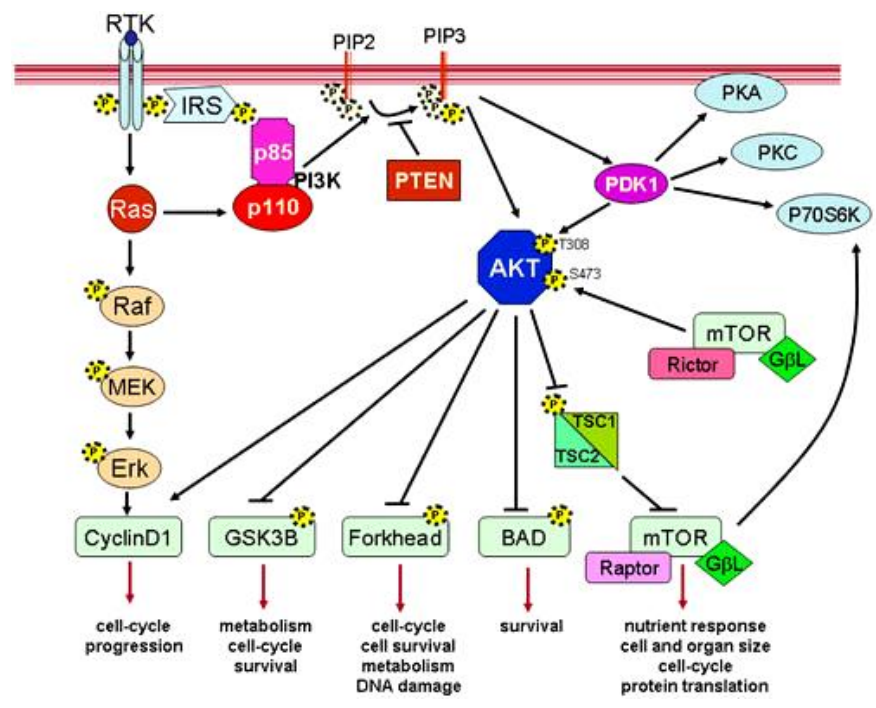

Fig 3: Event signaling process of P2P-SIP

ISSN: 0010-8189 
(1), (3) the user logs in at the client and sends a JXP publishing request to the local P2P extension module.

(2), (4)P2P expansion module sends JXTA message to JXTA network and issues announcement in the network.

User a is ready to initiate a call to user $\mathrm{B}$. first, user A sends a positioning request to the $\mathrm{P} 2 \mathrm{P}$ extension module on the local terminal, and broadcasts and sends JXTA messages to all users in the JXTA network. the query key contains the announcement of $1001 @$ room1.netsim. PeerClient on terminal b receives this message, finds that there is a notice of calling party's inquiry locally, and sends a TCP response message to P2P extension module of a, and the TCP message sent by B to a contains the published notice content of B. The Name field (1001@room1.netsim) is the URI in the form of B's user domain name, and the Desc field (1001@ 10.10.80.2) contains B's IP address. the extension module of a obtains the IP address of B's terminal from TCP message and directly initiates a call to B .. The signaling flow of call event is shown in Figure 4.

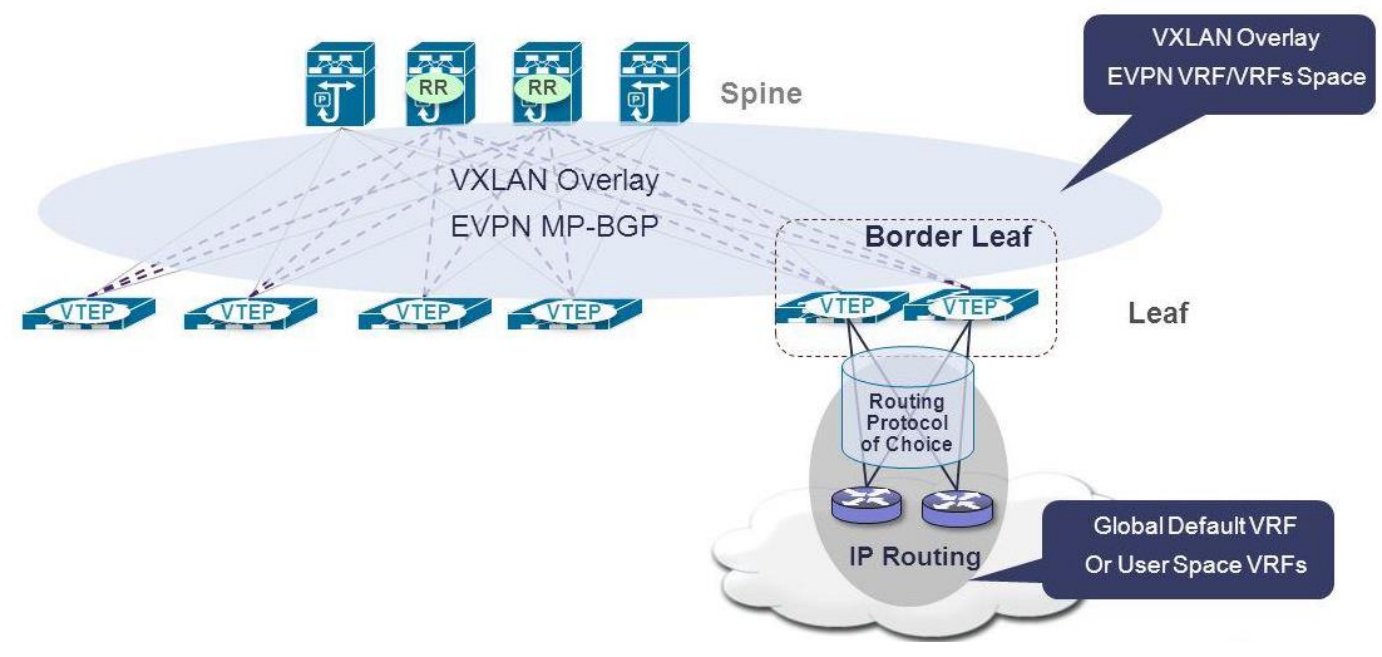

Fig 4: Signaling process of P2P-SIP call event

(1) peerClient of user a sends JXP location request to local P2P extension module.

(2)P2P extension module sends JXTA message to each user in JXTA network, and the query keyword Name is the announcement of $1001 @$ room1.netsim.

(3) P2P extension module of user B receives JXTA message and sends TCP ACK response to A.

(4) After receiving the response from B, the user A sends a positioning reply response to the client.

(5)-(11) After the user A gets the IP of B, he directly initiates a call to B in a back-to-back manner, and then the signaling flow is consistent with the traditional SIP.

\section{Conclusion}

In this paper, we implement a technical solution that combines P2P technology with SIP. On the open platform of JXTA, based on the open source SIP stack software package, we implement a P2P-SIP call system prototype by using the P2P publishing and location process instead of the standard SIP registration and search function. We have built a P2P-SIP prototype system on windows and Linux operating systems, realized the voice communication session function, and has the ability of cross platform. The clients running on windows and Linux can also communicate with each other. This paper provides a simple and comprehensive structure to combine $\mathrm{P} 2 \mathrm{P}$ and sip technology, and has openness and scalability, in order to develop and use more and newer technology to improve the P2P-SIP system.

ISSN: 0010-8189 


\section{Acknowledgements}

This research was supported by the key R \& D and Promotion Special Project of Henan Province (Grant No. 212102310086).

\section{References}

[1] Cai Zegao, Zhang Chunyue. Prototype Design and Implementation of P2P-SIP Softphone Based on Jxta. Journal of Nanjing University of Information Engineering: Natural Science Edition, 2010, 3:1217

[2] Wu Zhongxian, Zhou Jiang, Wang Wennai. Design and Implementation of P2p-sip. Telecommunication Science, 2009 (s2): 184-187

[3] Wang Zhe, Song Maoqiang. Design and Implementation of Network Resource Trading System Based on Jxta. Network Security Technology and Application, 2009, 10: 79-82

[4] Jiang Haoran, Xu Lin. Research and Design of P2p Online Shopping System Model Based on Jxta. Sci Tech Information, 2010, 35: 717-719

[5] Bi Jing. Research and Design of Cbm System Model Based on Jxta. Journal of Shenyang Institute of Aeronautical Technology, 2008, 25 (3): 49-49

[6] Wang Congming. Research and Implementation of Jxta Based Distributed System. Journal of Anhui University of Science and Technology, 2006, 20 (5): 29-32

[7] Zhou Jiang, Wu Zhongxian, Wang Wennai. Prototype Design of a P2p Sip Soft Phone Based on Jxta. Modern Telecommunication Technology, 2009, 2: 67-71

[8] Zhu, Chuan, Chang, Guiran, Ning, Wei. a Jxta-based Sip Communication System for P2p Wireless Networks. Wireless Communications Networking \&amp; Mobile Computing. Wicom.international Co, 2008:1-4.

[9] Fan Wei, Han Shuangxia, Ye Dongfen. Design of P2p Instant Messaging System Based on Jxta. Information and Computer (theoretical Edition), 2012 (05): 30-32

[10] Wang Zhe, Song Maoqiang. Design and Implementation of Network Resource Trading System Based on Jxta. Network Security Technology and Application, 2009 (10): 79-82

[11] Zhang Guowang, Shang Lina, Qi Chun. Architecture Design of Data Processing System Based on Hybrid P2p Network. Science Technology and Engineering, 2010 (18): 164-168 